\title{
Variabilidade espacial de cálcio, magnésio, fósforo, potássio no solo e produtividade da pimenta-do-reino
}

\author{
Eduardo 0. de J. Santos ${ }^{1}$, Ivoney G ontijo ${ }^{2} \&$ Lucas R. Nicole ${ }^{1}$
}

\begin{abstract}
RESU MO
A pimenta-do-reino é uma importante fonte de renda para o produtor rural, razão por que é uma espécie predominantemente cultivada por pequenos produtores porém ainda é pouco conhecida a relação da variação espacial dos nutrientes e da produtividade da cultura. O bjetivou-se, no presente trabalho, descrever a variabilidade espacial do $\mathrm{Ca}, \mathrm{Mg}, \mathrm{K}, \mathrm{P}$ no solo e a produtividade de uma lavoura de pimentado-reino, cultivada em um Latossolo Vermelho-Amarelo distrófico. 0 experimento foi conduzido em uma lavoura de pimenta-do-reino, no município de São Mateus, ES, plantada no espaçamento 3,0 x 1,8 m. 0 projeto foi instalado em uma malha retangular de $100 \times 120 \mathrm{~m}$. Em cada ponto amostral foram coletadas amostras de solo, na profundidade de $0-0,20 \mathrm{~m}$ e determinada a produtividade, totalizando 126 pontos, com distância mínima de $5 \mathrm{~m}$. O s dados foram submetidos à análise geoestatística. 0 bservouse estrutura de dependência espacial moderada e forte para os atributos químicos estudados. 0 estudo da variabilidade espacial dos atributos químicos do solo mostrou-se como ferramenta relevante na compreensão do comportamento dos nutrientes no solo podendo ser fundamental no auxílio à tomada de decisão que vise à maior produtividade da lavoura.
\end{abstract}

Palavras-chave: Piper nigrum L., atributos químicos, geoestatística

\section{Spatial variability of calcium, magnesium, phosphorus, potassium in soil and yield of black pepper}

\begin{abstract}
A B STRACT
The black pepper (Piper nigrum L.) has a social importance and it is mainly cultivated by small farmers, however the relationship between the spatial variability of nutrients in soil and yield of black pepper is little known. Thus, the purpose of this study was to analyse the spatial variability of $\mathrm{Ca}, \mathrm{Mg}, \mathrm{K}, \mathrm{P}$ in soil and black pepper yield, cultivated in a distrophic Red-Yellow Latosol. The study was carried out in a black pepper plantation, in São M ateus, in the State of Espírito Santo, Brazil. A $3 \times 1,8$ m spacing in a mesh of $100 \times 120 \mathrm{~m}$ (total area $12.000 \mathrm{~m}^{2}$ ), with 126 sampling points was used. Soil samples at depth of 0-0.2 $\mathrm{m}$ were collected at each point of the grid, in order to evaluate the soil chemical attributes. The variables presented a moderate and strong spatial dependence structure allowing their mapping by geostatistics techniques. Kriging maps were shown to be important tools for the understanding of spatial variability of soil nutrients, and it may be essential for decision making in the black pepper crop.
\end{abstract}

Key words: Piper nigrum L., chemical attributes, geostatistics 


\section{INTRODUÇÃO}

A pimenta-do-reino (Piper nigrum L.) é uma trepadeira originária da Índia, cultivada a pleno sol. O Brasil é um dos maiores produtores desta piperaceae, oscilando entre a segunda e terceira posição no mercado mundial. A produção brasileira de pimenta-do-reino, de acordo com pesquisas realizadas pelo IBGE (2010) atingiu, na safra 2009, cerca de 65 mil toneladas das quais cerca de $13 \%$ da produção provém do estado do Espírito Santo, explorando uma área superior a 2.300 ha.

O levantamento do índice de fertilidade do Estado do Espírito Santo, realizado por Pires et al. (2003), indicou que aproximadamente $89 \%$ dos solos capixabas foram classificados como de média a alta acidez potencial; este cenário, associado à exigência nutricional da pimenta-do-reino, revela a importância de estudos que indiquem a maneira mais eficiente de se realizar adubações nas referidas lavouras.

Estudos executados por Veloso \& Carvalho (1999) concluíram que a exigência de macronutrientes pela pimentado-reino segue a seguinte ordem decrescente: $\mathrm{N}>\mathrm{K}>\mathrm{Ca}>$ $\mathrm{Mg}>\mathrm{P}$. As quantidades totais de macronutrientes exportadas pelos frutos na colheita, são de: 11,$22 ; 6,15 ; 3,84 ; 1,18$ e 1,07 $\mathrm{kg} \mathrm{ha}^{-1}$ para, $\mathrm{N}, \mathrm{K}, \mathrm{Ca}, \mathrm{Mg}$ e $\mathrm{P}$, respectivamente, indicando, assim, a necessidade de fornecimento adequado de nutrientes capaz de suprir a exigência nutricional da pimenta-do-reino de modo a garantir a obtenção de altos níveis de produtividade.

O estudo da variabilidade espacial dos atributos químicos do solo sempre foi importante instrumento no processo da escolha da área experimental, locação das unidades experimentais, coleta de amostras e análise de produtividade (Silva \& Chaves, 2006), além da tomada de decisões sobre técnicas de manejo a serem adotadas porém a variabilidade espacial, se não considerada, pode levar a conclusões errôneas de resultados experimentais e ao uso incorreto de áreas agrícolas com finalidade comercial. Mas, se considerada, poderá tornar-se um instrumento significativo, possibilitando melhor conhecimento, planejamento e interpretação de experimentos usados para recomendações de manejo do solo e culturas (Albuquerque et al., 1996).

Lima et al. (2010a) verificaram, estudando a variabilidade espacial de atributos químicos do solo e a produção da cultura da pimenta-do-reino, que as técnicas de geoestatística se mostraram aplicáveis no diagnóstico das propriedades químicas do solo e da produção da pimenta-do-reino, permitindo seu mapeamento.

Propôs-se, neste trabalho, descrever a variabilidade espacial dos macronutrientes $\mathrm{Ca}, \mathrm{Mg}, \mathrm{P} \mathrm{e} \mathrm{K}$ e da produtividade de uma lavoura de pimenta-do-reino (Piper nigrum L.) no município de São Mateus, ES.

\section{Material e MÉtodos}

O experimento foi conduzido em uma lavoura de pimentado-reino da variedade Bragantina, plantada no espaçamento $3,0 \times 1,8 \mathrm{~m}\left(1.852\right.$ plantas ha $\left.^{-1}\right)$ em regime de irrigação por microaspersão, localizado no norte do estado do Espírito Santo, no município de São Mateus. As coordenadas geográficas são $18^{\circ} 41^{\prime} 58^{\prime \prime}$ de latitude Sul e $40^{\circ} 03^{\prime} 00^{\prime \prime}$ de longitude oeste com uma altitude média de $30 \mathrm{~m}$. O solo foi classificado como Latossolo Vermelho-Amarelo distrófico (EMBRAPA, 2006), textura argilo-arenosa, com teores de argila, silte e areia de 344, 113 e $543 \mathrm{~g} \mathrm{~kg}^{-1}$, respectivamente.

As análises de solo foram realizadas no Laboratório Agronômico de Análise de Solo, Folha e Água (LAGRO) do Centro Universitário Norte do Espírito Santo da Universidade Federal do Espírito Santo (UFES). Foi instalada uma malha retangular de $100 \times 120 \mathrm{~m}\left(12.000 \mathrm{~m}^{2}\right)$ com 126 pontos, com distância mínima de $5 \mathrm{~m}$ (Figura 1); para georreferenciamento da área foi utilizado um par de receptores GPS TechGeo ${ }^{\circledR}$, modelo GTR $\mathrm{G}^{2}$ geodésico cujos dados, após serem processados pela Rede Brasileira de Monitoramento Contínuo (RBMC) do IBGE, apresentaram precisão de $10 \mathrm{~mm}+1 \mathrm{ppm}$. Em cada ponto amostral foram coletadas amostras de solo na profundidade de 0-20 cm para análise química do solo, determinando-se os macronutrientes Cálcio, Magnésio, Fósforo e Potássio, de acordo com a EMBRAPA (1997). A produtividade foi estimada por meio da colheita das três plantas circunvizinhas a cada ponto amostral, sendo sua média multiplicada pela população de plantas por hectare (1.852 plantas ha $\left.^{-1}\right)$.

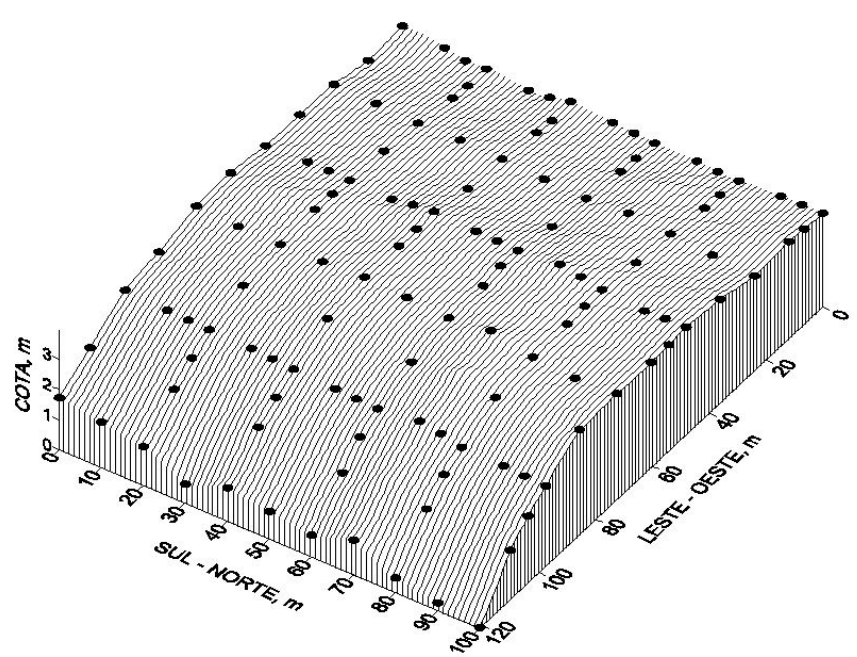

Figura 1. Modelo de el evação digital da área estudada com os respectivos locais de amostragem

Os resultados obtidos foram submetidos à análise exploratória dos dados, através da estatística descritiva, observadas as seguintes medidas: média aritmética mediana, variância amostral, desvio-padrão, coeficiente de variação, valores máximo e mínimo, coeficiente de assimetria e de curtose. A hipótese de normalidade dos dados foi testada pelo teste de Shapiro-Wilk a 5\% de probabilidade, por meio do programa estatístico Sisvar (Ferreira, 2000).

Os dados foram submetidos à análise geoestatística, com o objetivo de se definir o modelo de variabilidade espacial dos atributos do solo envolvidos neste estudo obtendo-se, assim, os semivariogramas e, posteriormente, o mapeamento de cada atributo químico estudado e da produtividade, através da krigagem. A análise da dependência espacial foi feita pela geoestatística com 
auxílio do programa computacional GS ${ }^{+}$Versão 7 (Gamma Design Software, 2004), que realiza os cálculos das semivariâncias amostrais cuja expressão pode ser encontrada em Vieira et al. (1983):

$$
\gamma(\mathrm{h})=\frac{\sum_{\mathrm{i}=1}^{\mathrm{n}(\mathrm{h})}\left[\mathrm{z}\left(\mathrm{x}_{\mathrm{i}}+\mathrm{h}\right) \mathrm{z}\left(\mathrm{x}_{\mathrm{i}}\right)\right]^{2}}{2 \mathrm{n}(\mathrm{h})}
$$

em que: $\mathrm{n}(\mathrm{h})$ número de pares amostrais $\left[\mathrm{z}\left(\mathrm{x}_{\mathrm{i}}\right) ; \mathrm{z}\left(\mathrm{x}_{\mathrm{i}}+\mathrm{h}\right)\right]$ separados pelo vetor $h$, sendo $z\left(x_{i}\right)$ e $z\left(x_{i}+h\right)$, valores numéricos observados do atributo analisado, para dois pontos $x_{i}$ e $x_{i}+h$, separados pelo vetor $\mathrm{h}$.

Os dados foram interpolados por meio da técnica da krigagem, a qual utiliza os parâmetros do semivariograma. Os modelos de semivariograma considerados foram o esférico e o exponencial. Em caso de dúvida entre mais de um modelo para o mesmo semivariograma, considerou-se só o maior valor do coeficiente de correlação obtido pelo método de validação cruzada e o menor valor da soma de quadrados do resíduo. Para elaboração dos mapas de distribuição espacial das variáveis foi utilizado o programa computacional Surfer (Golden Software, 1999). A razão de dependência espacial (GD), que é a proporção em percentagem do efeito pepita $(\mathrm{Co})$ foi calculada em relação ao patamar $(\mathrm{Co}+\mathrm{C})$, dada pela Eq. 2 :

$$
\mathrm{GD}=\frac{\mathrm{Co}}{\mathrm{Co}+\mathrm{C}} 100
$$

De acordo com Cambardella et al. (1994), GD apresenta a seguinte proporção: (a) dependência forte $<25 \%$; (b) dependência moderada de 25 a $75 \%$ e (c) dependência fraca $>75 \%$.

A classificação para interpretação de resultados de solo (Tabela 1), segundo Prezotti et al. (2007) para o estado do Espírito Santo, foi utilizada visando à confecção de mapas de variabilidade espacial baseados nas faixas estabelecidas por esses autores.

Tabela 1. Níveis para interpretação de resultados analíticos segundo a classificação de Prezotti et al. (2007)

\begin{tabular}{lccc}
\hline \multicolumn{1}{c}{ Variável } & Nível baixo & Nível médio & Nível alto \\
$\mathrm{Ca}\left(\mathrm{cmol}_{\mathrm{c}} \mathrm{dm}^{-3}\right)$ & $<1,5$ & $1,5-4,0$ & $>4,0$ \\
$\mathrm{Mg}\left(\mathrm{cmol}_{\mathrm{c}} \mathrm{dm}^{-3}\right)$ & $<0,5$ & $0,5-1,0$ & $>1,0$ \\
$\mathrm{P}\left(\mathrm{mg} \mathrm{dm}^{-3}\right)$ & $<10$ & $10-20$ & $>20$ \\
$\mathrm{~K}\left(\mathrm{mg} \mathrm{dm}^{-3}\right)$ & $<60$ & $60-150$ & $>150$ \\
\hline
\end{tabular}

\section{RESULTADOS E DISCUSSÃO}

Os resultados referentes à análise descritiva para os macronutrientes e a produtividade da cultura da pimenta-doreino, estão apresentados na Tabela 2. Os valores da média e da mediana para as variáveis magnésio e produtividade estão próximos, indicando distribuições simétricas e, para as demais variáveis, ocorreram valores com certo distanciamento entre média e mediana, passível de ser um indicativo de distribuição de dados assimétricos, em que as medidas de tendência central são dominadas por valores atípicos, concordando com os resultados de Mello et al. (2006); no entanto, somente a variável produtividade apresentou distribuição normal pelo teste de Shapiro-Wilk a $5 \%$.

Tabela 2. Estatística descritiva dos dados de cálcio, magnésio, fósforo, potássio e da produtividade da lavoura de pimenta-do-reino cultivada no município de São

\begin{tabular}{|c|c|c|c|c|c|}
\hline \multirow{2}{*}{$\begin{array}{l}\text { Estatística } \\
\text { descritiva }\end{array}$} & $\mathrm{Ca}$ & Mg & $\mathbf{P}$ & K & \multirow{2}{*}{$\begin{array}{l}\text { Produtividade } \\
\mathrm{kg} \mathrm{ha}^{-1}\end{array}$} \\
\hline & \multicolumn{2}{|c|}{$\mathrm{cmol}_{\mathrm{c}} \mathrm{dm}^{-3}$} & \multicolumn{2}{|c|}{$\mathrm{mg} \mathrm{dm}^{-3}$} & \\
\hline Média & 2,62 & 0,81 & 39,1 & 167 & 4161 \\
\hline Mediana & 2,50 & 0,79 & 32,5 & 160 & 4144 \\
\hline D.P & 1,05 & 0,22 & 26,5 & 49 & 1345 \\
\hline V.A & 1,10 & 0,05 & 702,9 & 2420,4 & 1809404 \\
\hline Mínimo & 0,36 & 0,36 & 3,3 & 83 & 1241 \\
\hline Máximo & 7,14 & 1,46 & 151,8 & 404 & 6946 \\
\hline C.V (\%) & 40,0 & 27,2 & 67,8 & 29,4 & 32,3 \\
\hline Ass. & 0,80 & 0,58 & 1,44 & 1,67 & $-0,18$ \\
\hline Curt. & 2,25 & 0,25 & 2,72 & 5,48 & $-0,65$ \\
\hline $\mathrm{p}$-valor $\times 10^{-4}$ & 6,985 & 36,615 & 0,0001 & 0,155 & $1392,54 *$ \\
\hline
\end{tabular}
Mateus, ES, obtidos a partir de 126 amostras

D.P - desvio padrão; V.A - variância amostral; C.V - coeficiente de variação; Ass. - coeficiente de assimetria; Curt. - coeficiente de curtose; Prod. - produtividade; *- distribuição normal pelo teste de Shapiro-Wilk a 0,05 de probabilidade

Mais importante que a normalidade dos dados é a ocorrência do efeito proporcional em que a média e a variância dos dados não sejam constantes na área de estudo, tendo em vista que os semivariogramas apresentam patamares bem definidos (Cavalcante et al., 2007).

O coeficiente de assimetria é uma medida utilizada para demonstrar como e quanto a distribuição de frequência se afasta da simetria: se o coeficiente de assimetria > 0, a distribuição é assimétrica à direita; se o coeficiente de assimetria $<0$, a distribuição é assimétrica à esquerda e, se o coeficiente de assimetria $=0$, a distribuiçãoé simétrica (Guimarães, 2000). Valores positivos de assimetria, conforme encontrados para $\mathrm{Ca}, \mathrm{Mg}, \mathrm{Pe}$ $\mathrm{K}$, indicam distribuição assimétrica à direita enquanto o valor negativo de assimetria para a produtividade da pimenta-do-reino sinaliza distribuição assimétrica à esquerda; já o coeficiente de curtose, que representa a dispersão, achatamento, da distribuição em relação a um padrão, geralmente a curva normal, em que: se o coeficiente de curtose $=0$ a distribuição é mesocúrtica, se o coeficiente de curtose $<0$ platicúrtica e se o coeficiente de curtose $>0$ leptocúrtica (Silva et al., 2010), todos os dados apresentaram distribuição lepticúrtica, com exceção da produtividade, com distribuição platicúrtica.

Os valores médios dos macronutrientes estudados (Tabela 2) foram classificados, de acordo com Prezotti et al. (2007), em teores médio para $\mathrm{Ca}\left(1,5-4,0 \mathrm{cmol}_{\mathrm{c}} \mathrm{dm}^{-3}\right)$ e $\mathrm{Mg}(0,5-1,0$ cmol $\left.\mathrm{dm}^{-3}\right)$ e elevados para oP $\left(>20 \mathrm{mg} \mathrm{dm}^{-3}\right)$ e $\mathrm{K}\left(>150 \mathrm{mg} \mathrm{dm}^{-3}\right)$ evidenciando, nas lavouras, as pesadas adubações desses elementos.

O coeficiente de variação foi considerado alto para fósforo e médio para as demais variáveis, de acordo com o critério de classificação proposto por Warrick \& Nielsen (1980). Resultados semelhantes foram obtidos por Cavalcante et al. (2007) referentes ao potássio, e por Lima et al. (2010b) referentes aos macronutrientes $\mathrm{Ca}, \mathrm{Ke} \mathrm{Mg}$.

Verificou-se dependência espacial em todos os atributos estudados, expressa por meio dos ajustes aos modelos de 
semivariogramas (Tabela 3 e Figura 2). O modelo que melhor se ajustou aos semivariogramas das variáveis cálcio, magnésio, potássio e à produtividade, foi o esférico, concordando com os resultados obtidos por Silva et al. (2007); para a variável fósforo, o melhor ajuste foi obtido com o modelo exponencial sendo que Cavalcante et al. (2007) obtiveram, estudando a variabilidade espacial desse atributo, resultado semelhante. Segundo Grego \& Vieira (2005) o modelo matemático esférico é o que predomina nos trabalhos em ciência do solo.

Tabela 3. M odelos e parâmetros estimados dos semivariogramas experimentais para cálcio, magnésio, fósforo, potássio e da produtividade da lavoura de pimenta-do-reino cultivada no município de São Mateus, ES

\begin{tabular}{|c|c|c|c|c|c|}
\hline \multirow{2}{*}{ Parâmetro } & $\mathrm{Ca}$ & Mg & $\mathbf{P}$ & K & \multirow{2}{*}{$\begin{array}{l}\text { Prod. } \\
\text { kg ha-1 }^{-1}\end{array}$} \\
\hline & \multicolumn{2}{|c|}{$\mathrm{cmol}_{\mathrm{c}} \mathrm{dm}^{-3}$} & \multicolumn{2}{|c|}{$\mathrm{mg} \mathrm{dm}^{-3}$} & \\
\hline Modelo & Esf. & Esf. & Exp. & Esf. & Esf. \\
\hline$E P\left(C_{0}\right)$ & 0,49 & 0,0158 & 278,0 & 1,0 & 537000 \\
\hline$P\left(C_{0}+C\right)$ & 1,03 & 0,0511 & 768,1 & 2192,0 & 2873693 \\
\hline$A(m)$ & 69,5 & 28,0 & 35,1 & 11,7 & 128,2 \\
\hline GD & 47,4 & 30,9 & 36,2 & 0,1 & 18,7 \\
\hline $\mathrm{R}^{2}$ & 0,93 & 0,96 & 0,79 & 0,82 & 0,94 \\
\hline SQR & 0,022 & $1,5 \times 10^{-5}$ & 14056 & 119741 & $5,8 \times 10^{11}$ \\
\hline
\end{tabular}

EP - efeito pepita; P - patamar; A - alcance; GD - razão de dependência es pacial; $R^{2}$ - coeficiente de determinação; SQR - soma de quadrado de resíduo; Esf. - esférico; Exp. - exponencial; Prod.produtividade

Com base na classificação de Cambardella et al. (1994) observa-se, na Tabela 3, que o potássio e a produtividade possuem dependência espacial forte; resultados semelhantes foram encontrados por Silva et al. (2008). Constatou-se dependência espacial moderada para os demais atributos, corroborando com os resultados observados por Corá et al. (2004) e Souza et al. (2004).

Observou-se que a produtividade apresentou o maior alcance (128,2 m) e entre os atributos químicos, foi do cálcio $(69,5 \mathrm{~m})$. Souza et al. (2004), atribuem este alto alcance do cálcio ao manejo do solo adotado através da calagem ou adubação, o que pode ter contribuído para aumentar o alcance caracterizando uma continuidade maior na distribuição desta variável. $\mathrm{O}$ alcance da dependência espacial é um atributo importante no estudo dos semivariogramas haja vista que representa a distância máxima em que os pontos amostrais estão correlacionados espacialmente entre si (Chaves \& Farias, 2009) ou seja, os pontos localizados numa área de raio igual ao alcance são mais homogêneos entre si do que com aqueles localizados fora desta área.

Pelos mapas da Figura 3 observa-se tendência geral de condições mais propícias ao desenvolvimento da lavoura a sudoeste da área, demonstradas pelas mais altas concentrações dos nutrientes estudados e consequentes maiores valores de produtividade nesta região da lavoura. Na região nordeste, onde se encontram as menores cotas, observam-se menores valores dos atributos químicos em estudo, principalmente cálcio e magnésio, podendo ser resultado de uma aplicação menor de calcário no solo, neste local da lavoura ou, ainda, uma perda desses nutrientes, provocada pelo processo erosivo do solo ocasionando menores taxas de produtividade de pimenta-doreino nesta região específica (Figura 3E). O cálcioé um nutriente com papel preponderante no crescimento radicular das plantas
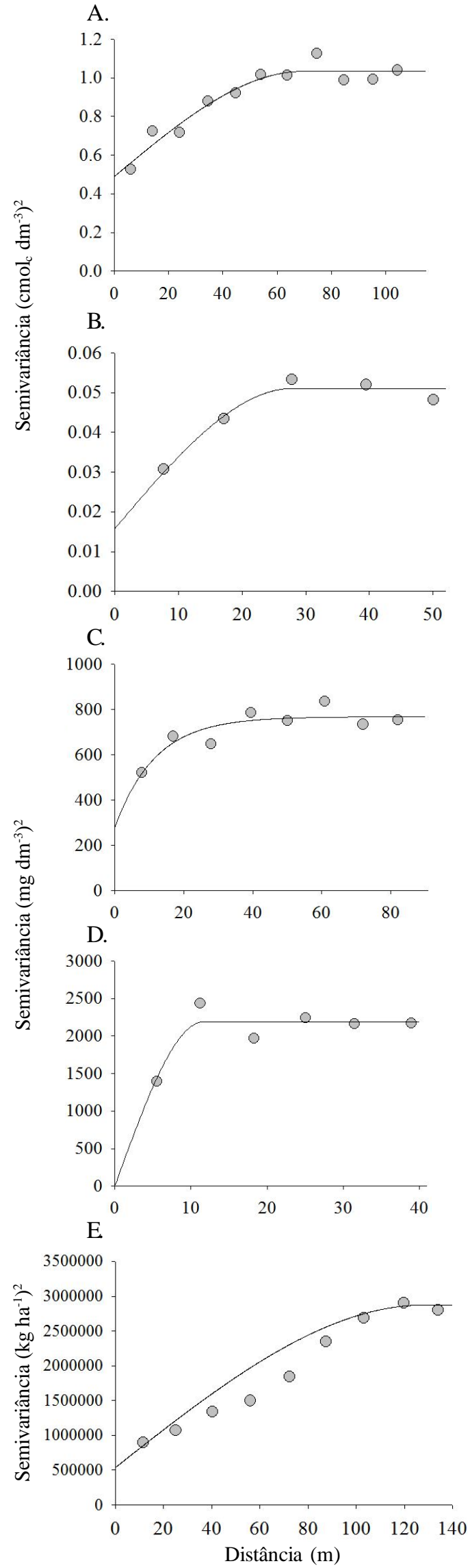

Figura 2. Modelos de semivariogramas ajustados para cálcio $(A)$, magnésio $(B)$, fósforo $(C)$, potássio $(D)$ e produtividade (E) 
A.

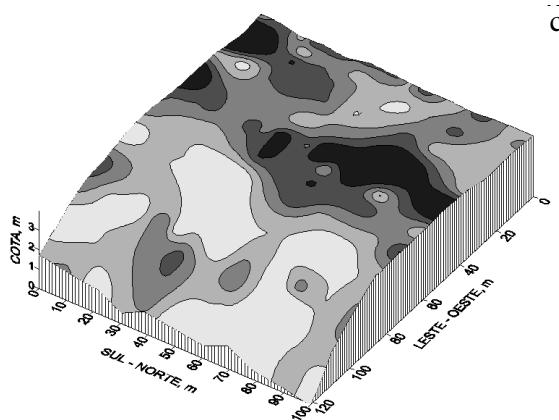

B.

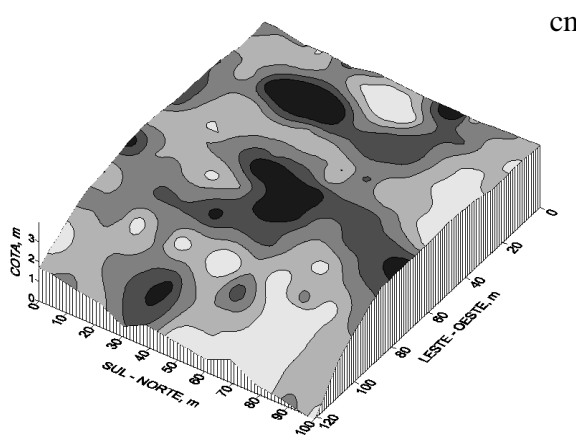

C.

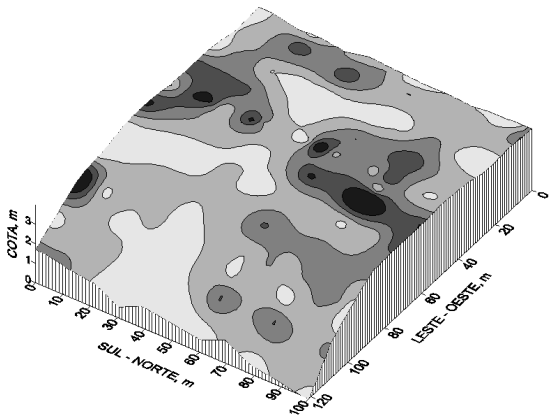

D.

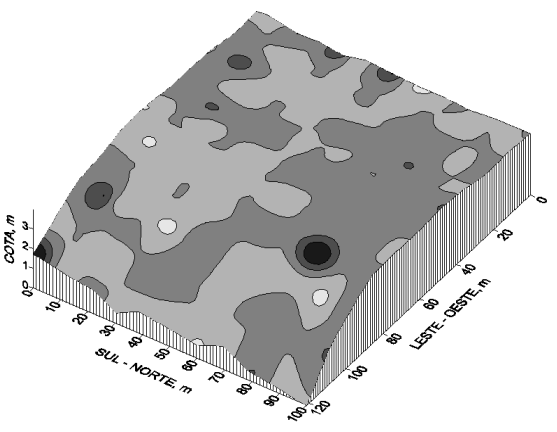

E

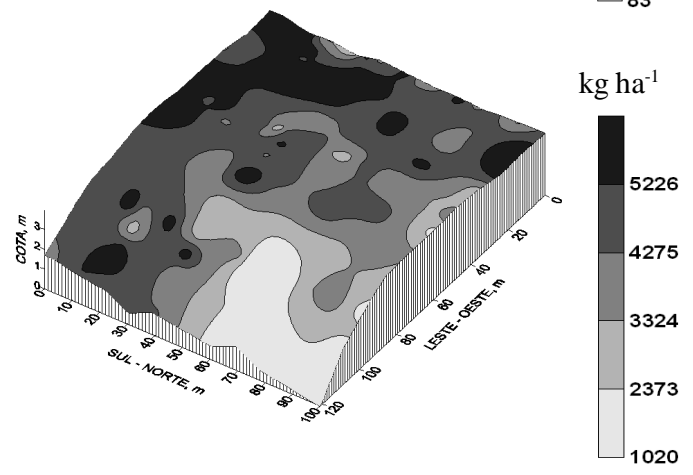

Figura 3. Mapas de isolinhas das variáveis cálcio (A), magnésio $(B)$, fósforo $(C)$, potássio $(D)$ e produtividade da pimenta-do-reino (E), representados conjuntamente com o relevo da área

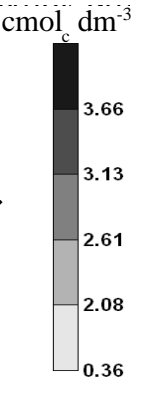

cmol $\mathrm{dm}^{-3}$
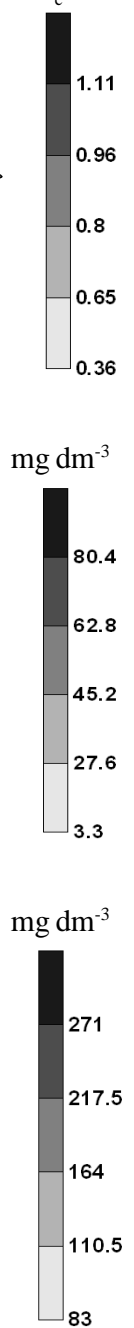

26

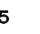

4

3

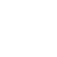


de nível médio para cálcio e magnésio, com pequenas áreas com nível baixo nas regiões de menor cota (Figura 4 A e B) e predominância do nível alto para o fósforo e potássio (Figura 4 $\mathrm{C}$ e D) demonstrando claramente zonas homogêneas na área em estudo, podendo servir para delimitar zonas de manejo diferenciadas para fertilidade do solo. Barbieri et al. (2008) observaram que as formas do relevo demonstraram ser indicadoras de zonas específicas visando à aplicação de insumos a taxas variáveis. Para Molin (2002) a definição de unidades de manejo do solo que consigam representar regiões homogêneas quanto aos atributos que influenciam o desenvolvimento das culturas, é uma das etapas mais desafiadoras no contexto de agricultura de precisão.

\section{CONClusÕES}

1. Verifica-se dependência espacial para todos os atributos químicos estudados, com grau de dependência espacial moderado e forte.

2. Observa-se o maior alcance de dependência espacial para produtividade da pimenta-do-reino $(128,2 \mathrm{~m})$ e o menor para o potássio $(11,7 \mathrm{~m})$.

3. O estudo da variabilidade espacial dos atributos químicos do solo pela geoestatística utilizando-se a técnica da krigagem, desponta como ferramenta imprescindível na compreensão do comportamento dos nutrientes no solo, podendo ser fundamental no auxílio à tomada de decisão que visem maior produtividade da lavoura de pimenta-do-reino.

\section{Agradecimentos}

Os autores agradecem a Eliseu Bonomo, pela cessão da área de estudo, e à FAPES, pela concessão de bolsa ao primeiro autor.

\section{LITERATURA CITADA}

Albuquerque, J. A.; Reinert, D. J.; Firorin, J. E. Variabilidade de solo e planta em Podzólico Vermelho-amarelo. Revista Brasileira de Ciência do Solo, v.20, p.151-157, 1996.

Barbieri, D. M.; Marques Júnior, J.; Pereira, G. T. Variabilidade espacial de atributos químicos de um Argissolo para aplicação de insumos à taxa variável em diferentes formas de relevo. Engenharia Agrícola, v.28, p.645-653, 2008.

Cambardella, C. A. Moornam, T. B.; Novak, J. M.; Parkin, T. B.; Karlen, D. L.; Turco, R. F.; Konopka, A. E. E. Field-scale variability of soil properties in central Iowa soils. Soil Science Society of America Journal, v.58, p.1501-1511, 1994.

Cavalcante, E. G. S.; Alves, M. C.; Pereira, G. T.; Souza, Z. M. Variabilidade espacial de MO, P, K e CTC do solo sob diferentes usos e manejos. Ciência Rural, v.37, p.394-400, 2007.

Chaves, L. H. G.; Farias, C. H. A. Variabilidade espacial de cobre e manganês em Argissolo sob cultivo de cana-de-açúcar. Revista Ciência Agronômica, v.40, p.211-218, 2009.
Corá, J. E.; Araújo, A. V.; Pereira, G. T.; Beraldo, J. M. G. Variabilidade espacial de atributos do solo para adoção do sistema de agricultura de precisão na cultura de canade-açúcar. Revista Brasileira de Ciência do Solo, v.28, p.1013-1021, 2004.

EMBRAPA - Empresa Brasileira de Pesquisa Agropecuária. Centro Nacional de Pesquisa de Solos (Rio de Janeiro, RJ). Manual de métodos de análise de solo. 2.ed. Rio de Janeiro: Embrapa CNPS, 1997.212p.

EMBRAPA - Empresa Brasileira de Pesquisa Agropecuária. Sistema brasileiro de classificação de solos. 2. ed. Brasília: Embrapa CNPS, 2006.306p.

Ferreira, D. F. Sisvar. Sistema para análise de variância, para Windows versão 4.3. Lavras: UFLA/Departamento de Ciências Exatas, 2000.

Gamma Design Software. Geoestatistics for the environmental sciences. Version 7.0. Michigan, 2004. CD Rom.

Golden Software. Surfer for windows: Realise 7.0: Contouring and 3D surface mapping for scientist's engineers user's guide. New York: Golden Software, 1999. 619p.

Grego, C. R.; Vieira, S. R. Variabilidade espacial de propriedades físicas do solo em uma parcela experimental. Revista Brasileira de Ciência do Solo, v.29, p.169-177, 2005.

Guimarães. E. C. Variabilidade espacial de atributos de um Latossolo Vermelho-escuro textura argilosa da região do cerrado, submetido ao plantio direto e ao plantio convencional. Campinas: UEC, 2000. 85p. Tese Doutorado

IBGE - Instituto Brasileiro de Geografia e Estatística. Agricultura. http://www.sidra.ibge.gov.br/bda/agric/default.asp?t=2\&z=t $\& o=11 \& u 1=1 \& u 2=1 \& u 3=1 \& u 4=1 \& u 5=1 \& u 6=1$. 4 Mar. 2010.

Lima, J. S. S.; Oliveira, R. B.; Rocha, W.; Oliveira, P. C.; Quartezani, W.Z. Análise espacial de atributos químicos do solo e da produção da cultura de pimenta-do-reino. Idesia, v.28, p.31-39, 2010a.

Lima, J. S. S.; Souza, G. S.; Silva, S. A. Amostragem e variabilidade espacial de atributos químicos do solo em área de vegetação natural em regeneração. Revista Árvore, v.34, p.127-136, $2010 \mathrm{~b}$.

Mello, G.; Bueno, C. R. P.; Pereira, G. T. Variabilidade espacial das propriedades físicas e químicas do solo em áreas intensamente cultivadas. Revista Brasileira de Engenharia Agrícola e Ambiental, v.10, p.294-305, 2006.

Molin, J. P. Definição de unidades de manejo a partir de mapas de produtividade. Engenharia Agrícola, v.22, p.83-92, 2002.

Pires, F. R.; Caten, A.; Martins, A. G.; Esposti, M. D. D. Levantamento da fertilidade nas principais unidades de mapeamento do Espírito Santo. Revista Ciência Agronômica, v.34, p.115-123, 2003.

Prado, R. M.; Natale, W. Calagem na nutrição de cálcio e no desenvolvimento do sistema radicular da goiabeira. Pesquisa Agropecuária Brasileira, v.39, p.1007-1012, 2004.

Prezotti, L. C.; Gomes, J. A.; Dadalto, G. G.; Oliveira, J. A. Manual de Recomendação de Calagem e Adubação para o Estado doEspírito Santo - $5^{\mathrm{a}}$ aproximação. Vitória: SEEA/INCAPER/ CEDAGRO, 2007.305p.

Ritchey, K. D.; Silva, J. E.; Costa, U. F. Calcium deficiency in clayey B horizons of savanna oxisols. Soil Science, v.133. p.378-382, 1982. 
Silva, F. M.; Souza, Z. M.; Figueiredo, C. A. P.; Marques Júnior, J.; Machado, R. V. Variabilidade espacial de atributos químicos e de produtividade na cultura do café. Ciência Rural, v.37, p.401-407, 2007.

Silva, F. M.; Souza, Z. M.; Figueiredo, C. A. P.; Viera, L. H. S.; Oliveira, E. Variabilidade espacial de atributos químicos e produtividade da cultura do café em duas safras agrícolas. Ciência Agrotécnologica, v.32, p.231-241, 2008.

Silva, P. C. M.; Chaves, L. H. G. Avaliação da variabilidade espacial de atributos químicos de um solo do perímetro irrigado de Petrolina, PE. Agropecuária Técnica, v.27, p.5-12, 2006.

Silva, S. A.; Lima, J. S. S.; Xavier, A. C.; Teixeira, M. M. Variabilidade espacial de atributos químicos de um Latossolo Vermelho-amarelo húmico cultivado com café. Revista Brasileira de Ciência do Solo, v.34, p.15-22, 2010.
Souza, Z. M.; Marques Júnior, J.; Pereira, G. T.; Moreira, L. F. Variabilidade espacial do $\mathrm{pH}, \mathrm{Ca}, \mathrm{Mg}$ e $\mathrm{V} \%$ do solo em diferentes formas do relevo sob cultivo de cana-de-açúcar. Ciência Rural, v.34, p.1763-1771, 2004.

Veloso, C. A. C.; Carvalho, E. J. M. Absorção e extração de alguns nutrientes pela cultivar 'Guajarina' de pimenta-doreino. Scientia Agricola, v.56, p.443-447, 1999.

Vieira, S. R.; Hatfield, J. L.; Nielsen, D. R.; Biggar, J. W. Geostatistical theory and application to variability of some agronomical properties. Hilgardia, v.51, p.1-75, 1983.

Warrick, A. W.; Nielsen, D. R. Spatial variability of soil physical properties in the field. In: HILLEL, D. (ed.). Applications of soil physics. New York: Academic Press, 1980. Cap.2, p.319344. 